\title{
Shadow poles in coupled-channel problems calculated with Berggren basis
}

\author{
R. M. Id Betan, ${ }^{1,2}$ A.T. Kruppa ${ }^{3}$ and T. Vertse ${ }^{3,4}$ \\ ${ }^{1}$ Physics Institute of Rosario (CONICET), Bv. 27 de Febrero 210 bis, S2000EZP Rosario, Argentina \\ ${ }^{2}$ Department of Physics and Chemistry FCEIA(UNR), \\ Av. Pellegrini 250, S2000BTP Rosario, Argentina \\ ${ }^{3}$ Institute for Nuclear Research Hungarian Academy of Sciences (ATOMKI), PO Box 51, H-4001, Debrecen, Hungary \\ ${ }^{4}$ University of Debrecen, Faculty of Informatics, PO Box 12, H-4010, Debrecen, Hungary
}

\begin{abstract}
Background: In coupled-channel models the poles of the scattering S-matrix are located on different Riemann sheets. Physical observables are affected mainly by poles closest to the physical region but sometimes shadow poles have considerable effect, too.

Purpose: The purpose of this paper is to show that in coupled-channel problems all poles of the S-matrix can be located by an expansion in terms of a properly constructed complex-energy basis.

Method: The Berggren basis is used for expanding the coupled-channel solutions.

Results: The locations of the poles of the S-matrix for the Cox potential, constructed for coupled channel problems, were numerically calculated and compared with the exact ones. In a nuclear physics application the $J^{\pi}=3 / 2^{+}$resonant poles of ${ }^{5} \mathrm{He}$ were calculated in a phenomenological two channel model. The properties of both the normal and shadow resonances agree with previous findings.

Conclusions: We have shown that, with an appropriately chosen Berggren basis, all poles of the S-matrix including the shadow poles can be determined. We have found that the shadow pole of ${ }^{5} \mathrm{He}$ migrates between Riemann sheets if the coupling strength is varied.

PACS numbers: 21.60.-n,21.60.Cs,02.60.-x
\end{abstract}

\section{INTRODUCTION}

The physics of coupled-channel (CC) models spans many research areas, ranging from traditional nuclear physics [1 3] to atomic physics [4, 5]. It may be extended to chiral perturbation theory combined with a multichannel approach [6 8], and to hypernuclear physics [9].

As in scattering theory in general, in scattering $\mathrm{CC}$ models the exploitation of the analytic properties of the $\mathrm{S}$-matrix is a basic tool in describing the scattering processes [10]. The S-matrix as a function of energy is analytic over a Riemann surface of many sheets [11 13]. The analytic continuation of the physical S-matrix may have poles on the unphysical sheets. These poles correspond to the eigenvalues of the Hamiltonian with various special boundary conditions. The eigenfunctions belonging to these generalized eigenvalues are referred as resonance/virtual states of the system. On the physical sheet the eigenfunctions are asymptotically decreasing, i.e., they are bound states. The asymptotic forms of the resonance/virtual states are not bounded. In the following the term resonant state will be used for all discrete (bound, resonance and virtual) states.

The main question in a CC scattering model is how the S-matrix poles affect the scattering observables. Obviously, they are affected by poles closest to the physical region. However, it was recognized long ago that poles far from the physical regions (so called shadow poles) may have large effects on observables [14, 15]. This phenomenon occurs in atomic [16 19], nuclear [20 23] and particle physics [24 26] as well. The effect of the shadow poles was also studied for two-dimensional confined electron gas [27].
As for the finding of all types of poles of the S-matrix i.e. solving the $\mathrm{CC}$ equation with a generalized asymptotic condition there are two approaches: either the expansion is modified so as to allow for the special boundary conditions, and the problem is solved with orthodox methods or the problem is transformed so that the usual expansion may provide the modified solutions.. The most straightforward example for the second approach is the complex scaling method, which has a long history [28, 29]. In a recent nuclear three-body application [30] the backrotation problem of this method has also been solved. The usage of the complex scaling method to CC problems sometimes requires slight extensions, e.g. when shadow poles are sought [31].

An approach of the first kind nowadays often used is the series expansion of the wave function on an extended basis. The basis includes the bound states, selected resonance/virtual states as well as complex energy scattering states of the potential [32]. The existence of such a basis was proven by T. Berggren [33]. Berggren bases were used in the solution of many-body shell model calculations [34, 35], especially when weakly bound or unbound states of certain nuclei observed in radioactive beam facilities were to be described by Gamow shell model or complex-energy shell model [36]. In certain applications [37, 38] it was shown that anti-bound states can also be included as basis states.

The Berggren bases were also used in CC problems: the radial coupled HFB equations [39] and the CC Lane equation describing isobaric analog states [40] were solved with it.

The aim of this paper is to show that in CC problems all poles of the S-matrix can be located with properly 
constructed Berggren bases. To this end, we reproduce the results of an exactly solvable $\mathrm{CC}$ problem: the one with the Cox potential. This CC problem can be solved exactly [41 44] so there is no ambiguity in the location of the S-matrix poles. The analytic solution of the Cox potential offers a unique opportunity for testing the results of the numerical solution of the CC problem.

Another CC problem we consider is the description of the $\frac{3}{2}^{+}$resonance state of the nucleus ${ }^{5} \mathrm{He}$. This resonance is famous due to its role in the production of thermonuclear energy and in primordial stellar nucleosynthesis. A phenomenological analysis [20] revealed the presence of a shadow pole. The strong $t+d \rightarrow \alpha+n$ transition is accounted for the presence of this pole. The structure of the $\frac{3}{2}^{+}$state is investigated for example in the works [21, 45 47]. Here we apply a simple phenomenological CC model in order to show a physical situation where a shadow pole plays an important role and can be explored using the Berggren basis technique.

The paper is organized as follows. Section $\amalg$ describes the CC Cox problem and its analytic solution. In Section III we discuss how to use the Berggren basis for the solution of a CC problem. Here we discuss the main point of the paper: how to choose the Berggren basis in order to get all poles of the S-matrix. Sections IV and V are dedicated to the applications. The first one presents the results for the $\mathrm{CC}$ Cox problem, both in the analytic framework and in numerical frameworks, while the second one contains our study of the pole structure of the $J^{\pi}=3 / 2^{+}$state of ${ }^{5} \mathrm{He}$ in a two-channel approximation. Finally, the conclusions are contained in Section. VI]

\section{THE COX COUPLED-CHANNEL PROBLEM}

\section{A. The Cox potential}

The two-channel radial Schrödinger-equation with energy $E$ in reduced units reads

$$
H \psi(r, E)=K^{2} \psi(r, E),
$$

where $K=\operatorname{diag}\left(k_{1}, k_{2}\right), k_{i}=\sqrt{E-\Delta_{i}}$ denotes the channel wave numbers, $\Delta_{i}$ are the threshold energies. We will use as in [42] $\Delta_{1}=0$ and $\Delta_{2}=\Delta>0$. The notation $\operatorname{diag}\left(a_{1}, a_{2}\right)$ means a two by two diagonal matrix with elements $a_{i}$ in the main diagonal. The Hamiltonian is

$$
H=\left(\begin{array}{cc}
-\frac{d^{2}}{d r^{2}}+v_{11}(r) & v_{12}(r) \\
v_{21}(r) & -\frac{d^{2}}{d r^{2}}+v_{22}(r)
\end{array}\right)
$$

and the solution forms a vector

$$
\psi=\left(\begin{array}{l}
\psi_{1} \\
\psi_{2}
\end{array}\right) .
$$

The Schrödinger equation in Eq. (1) has two matrix value Jost solution from which the Jost matrix can be constructed defining both the scattering and bound state solutions. We classify the solutions of Eq. (11) as it is done in Ref. [42]. We call a solution a bound state when the zero of the determinant of the Jost matrix $k_{1}$ and $k_{2}$ are both pure positive imaginary numbers. We call the solution a virtual state or anti-bound state, when the zero of the Jost-matrix determinant corresponds to a real energy below the thresholds and the zero is lying on the imaginary $k_{i}$ axes, but not all of them are located on the positive imaginary axis. Finally, we call the solution a resonance if the zero is not lying on any of the imaginary $k_{i}$ axes, hence the corresponding energy is complex or if real then it is above at least one of the thresholds.

The derivation of the Cox potential and how to solve it exactly are given in [42 44]. To make the paper selfcontained we collect some formulas. The Cox [41] interaction matrix

$$
V(r)=\left(\begin{array}{ll}
v_{11}(r) & v_{12}(r) \\
v_{21}(r) & v_{22}(r)
\end{array}\right) .
$$

is given by

$$
V(r)=-\mathcal{K}+2 \mathcal{K}^{1 / 2}(I+X(r))^{-1} \mathcal{K}^{1 / 2},
$$

where $I$ is the $2 \times 2$ unit matrix and

$$
\begin{aligned}
& X(r)=\operatorname{diag}\left(\exp \left(-\kappa_{1} r\right), \exp \left(-\kappa_{2} r\right)\right) X_{0} \\
& \quad \times \operatorname{diag}\left(\exp \left(-\kappa_{1} r\right), \exp \left(-\kappa_{2} r\right)\right) .
\end{aligned}
$$

The symmetric $2 \times 2$ matrix $X_{0}$ contains the parameters of the potential. The factorization wave numbers $\kappa_{1}$ and $\kappa_{2}$ are positive parameters and they satisfy the condition $\kappa_{2}^{2}-\kappa_{1}^{2}=\Delta$. (we will use $\kappa_{1}=\kappa$ as independent parameter). The matrix $\mathcal{K}$ is related to the factorization energies by $\mathcal{K}=\operatorname{diag}\left(\kappa_{1}, \kappa_{2}\right)$. Of course, the interaction matrix (4) is symmetric.

The determinant of the Jost matrix is given by [42]

$$
f(k, p)=\frac{\left(k+i \alpha_{1}\right)\left(p+i \alpha_{2}\right)+\beta^{2}}{\left(k+i \kappa_{1}\right)\left(p+i \kappa_{2}\right)} .
$$

Here we denote for convenience the channel wave numbers as $k_{1}=k$ and $k_{2}=p$. The threshold condition reads $k^{2}-p^{2}=\Delta$. The zeros of the function $f(k, p)$ determine the position of the bound, virtual and resonance states. Interestingly the Cox potential depends on the factorization wave numbers but the eigenenergies are independent from $\kappa_{i}$.

The connection between the parametrization $\alpha_{1}, \alpha_{2}, \beta$ and $X_{0}$ is given by the equation

$$
U_{0}=\left(\begin{array}{cc}
\alpha_{1} & \beta \\
\beta & \alpha_{2}
\end{array}\right)=\mathcal{K}^{1 / 2}\left(I-X_{0}\right)\left(I+X_{0}\right)^{-1} \mathcal{K}^{1 / 2}
$$

and the inverse relation is

$$
X_{0}=\mathcal{K}^{-1 / 2}\left(\mathcal{K}-U_{0}\right)\left(\mathcal{K}+U_{0}\right)^{-1} \mathcal{K}^{1 / 2} .
$$




\section{B. Reduced inverse problem}

In a direct problem we calculate the eigenenergies and the corresponding channel wave numbers $\left(k_{i}, p_{i}\right)$ of the Cox CC equations for a given set of potential parameters $\alpha_{1}, \alpha_{2}, \beta$, and $\Delta$. In the inverse problem we fix a few eigenenergies or other characteristics of the problem and search for the parameters of the potential which give back the fixed characteristics.

A very convenient approach was introduced in Ref. [48]. In this approach one first fixes two solutions and the other two solutions are obtained in closed form. Let us fix $\left(k_{1}, p_{1}\right)$ and $\left(k_{2}, p_{2}\right)$ as the first two zeros of the Jost-matrix determinant $f(k, p)$ then from (7) we get

$$
\begin{aligned}
& \left(k_{1}+i \alpha_{1}\right)\left(p_{1}+i \alpha_{2}\right)+\beta^{2}=0 \\
& \left(k_{2}+i \alpha_{1}\right)\left(p_{2}+i \alpha_{2}\right)+\beta^{2}=0
\end{aligned}
$$

and we obtain the parameters $\alpha_{1}$ and $\alpha_{2}$ in terms of $\left(k_{1}, p_{1}\right)$ and $\left(k_{2}, p_{2}\right)$ and $\beta$

$$
\begin{aligned}
& \alpha_{1}=\frac{1}{2}\left[i\left(k_{1}+k_{2}\right) \pm \sqrt{-\Delta_{k}^{2}-4 \beta^{2} \frac{\Delta_{k}}{\Delta_{p}}}\right] \\
& \alpha_{2}=\frac{1}{2}\left[i\left(p_{1}+p_{2}\right) \mp \sqrt{-\Delta_{p}^{2}-4 \beta^{2} \frac{\Delta_{p}}{\Delta_{k}}}\right],
\end{aligned}
$$

where $\Delta_{k}=k_{2}-k_{1}$ and $\Delta_{p}=p_{2}-p_{1}$. The signs define two sets of solutions which correspond to the same $\left(k_{1}, p_{1}\right)$ and $\left(k_{2}, p_{2}\right)$ but two different $\left(k_{3}, p_{3}\right)$ and $\left(k_{4}, p_{4}\right)$ roots. In selecting the solution either the upper or the lower signs are to be taken.

The other two roots $\left(k_{3}, p_{3}\right)$ and $\left(k_{4}, p_{4}\right)$ are determined in Ref. [48] as follows.

$$
\begin{aligned}
& k_{3}=\frac{1}{2}\left[\mp i \sqrt{-\Delta_{k}^{2}-4 \beta^{2} \frac{\Delta_{k}}{\Delta_{p}}}+\sqrt{D_{k}}\right] \\
& p_{3}=\frac{1}{2}\left[\mp i \sqrt{-\Delta_{p}^{2}-4 \beta^{2} \frac{\Delta_{p}}{\Delta_{k}}}+\sqrt{D_{p}}\right] \\
& k_{4}=\frac{1}{2}\left[\mp i \sqrt{-\Delta_{k}^{2}-4 \beta^{2} \frac{\Delta_{k}}{\Delta_{p}}}-\sqrt{D_{k}}\right] \\
& p_{4}=\frac{1}{2}\left[\mp i \sqrt{-\Delta_{p}^{2}-4 \beta^{2} \frac{\Delta_{p}}{\Delta_{k}}}-\sqrt{D_{p}}\right],
\end{aligned}
$$

where $D_{k}=\Delta_{k}^{2}+4 \beta^{2} \frac{\Delta_{p}}{\Delta_{k}}+4 k_{1} k_{2}$ and $D_{p}=\Delta_{p}^{2}+4 \beta^{2} \frac{\Delta_{k}}{\Delta_{p}}+$ $4 p_{1} p_{2}$. Note the difference in sign of the second term in $p_{3}$ and $p_{4}$ with respect to Eq. (50) in Ref. [48].

\section{SOLUTION USING BERGGREN BASIS}

We calculate the eigenvalues of the CC Cox problem by diagonalizing the Hamiltonian (2) in the Berggren bases of the potentials $v_{11}(r)$ and $v_{22}(r)$. We consider two auxiliary problems

$$
\left[-\frac{d^{2}}{d r^{2}}+v_{i i}(r)-E_{n}^{(i)}\right] u_{n}^{(i)}(r)=0 \quad i=1,2 .
$$

The states of the Berggren basis are the solutions of Eq. (18) and for each channel they are composed of the resonant basis states which are eigensolutions of Eq. (18) with purely outgoing wave boundary condition, i.e. they correspond to the poles of the $S$-matrix in that channel. The resonant states (bound,virtual and resonance solutions) with energy $E_{n}^{(i)}$ are denoted by $u_{n}^{(i)}(r)$. Beside the resonant states the basis contains scattering states along a complex contour $L$. The scattering solutions are denoted by $u^{(i)}(r, E)$ or $u^{(i)}(r, k)$ if we use the energy $E$ or wave number $k$, respectively.

The shape of the contour $L$ is restricted by certain rules 33 . In the $k$ plane the contour has to go through the origin and has to be symmetric to the origin, i.e. if $k$ is on the contour $L$ then $-k$ should be on the contour $L$ too. Contour $L$ is divided by the origin to two halfs, denoted by $L^{+}$and $L^{-}$. The half $L^{+}$is between $k=0$ and $k \rightarrow+\infty$ with $\operatorname{Im}(k)<0$ in Figure 1 and Figure 2 . The $L^{+}$part for large $|k|$ values has to go back to the real axis and remain there. The contribution of the $L^{-}$half is equal to the one of $L^{+}$and the factor 2 is included in the normalization of the scattering wave functions. When the contour is the full real $k$ line similar decomposition of the $k$ values is used in [49] proving the completeness of the scattering states.

In the set up of the basis only those resonant states have to be included into the Berggren basis whose wave numbers are in the shaded area, i.e. between the contour $L^{+}$and the positive $k$-axis. Similar relations should be hold for the $p$ contour of the second channel. (See Figures 1 and 2 the wave numbers to be included into the basis should be also in the shaded area of the figures.)

The completeness relation of the Berggren basis reads

$$
\begin{gathered}
\delta\left(r-r^{\prime}\right)=\sum_{n=b, d, v} u_{n}^{(i)}(r) u_{n}^{(i)}\left(r^{\prime}\right) \\
\quad+\int_{L^{+}} d k u^{(i)}(r, k) u^{(i)}\left(r^{\prime}, k\right) .
\end{gathered}
$$

In this relation (and later) the notation $n=b, d, v$ means that the sum over $n$ runs through all bound states, decaying resonances and virtual states in the shaded area of Fig. 1, The integral in Eq. (19) is over the scattering states along the $L^{+}$half contour. The completeness relation in Eq. (19) for chargeless particles was introduced in Ref. 33] and its validity has been shown for charged particles too 50 52.

Since we can not handle the continuum part exactly the complex contour is discretized and truncated in order to have a finite number of contour states. The renormalization of the discretized contour states was introduced first in Ref. [53] and it is performed as in Ref. [36]. We use as discretization points $E_{k}^{(i)}\left(k=1, \ldots, N_{i}\right)$ the abscissas 


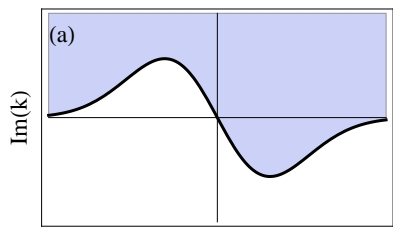

$\operatorname{Re}(\mathrm{k})$
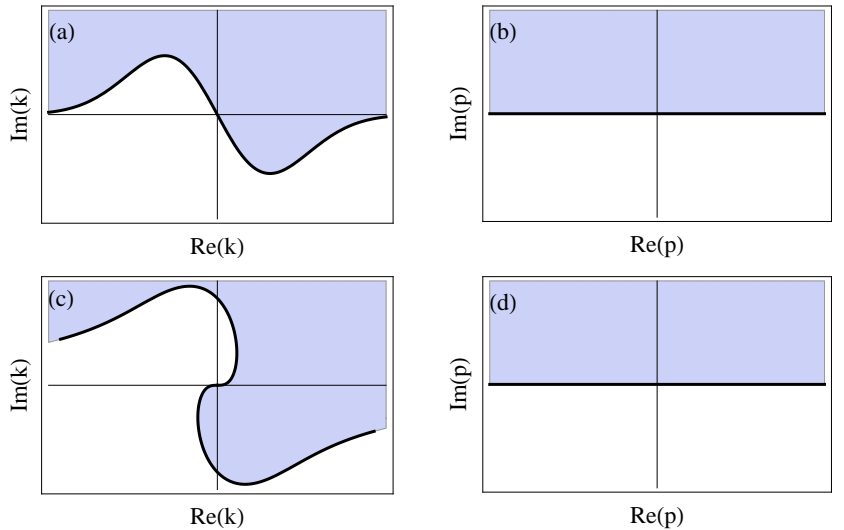

FIG. 1. (Color online) Illustration of the contours $L$ (thick lines) on the $k$ and $p$ planes for the second Riemann sheet. Resonance states can be determined with contours similar to the parts (a) and (b). Both virtual and resonance states can be calculated with contours similar to the parts (c) and (d). The double roles of the shaded areas are explained in the text.

of a Gaussian quadrature procedure. The corresponding weights of the quadrature points are denoted by $h_{k}^{(i)}$. After discretizing the integral in Eq. (19) an approximate completeness relation for the finite number of basis states reads

$$
\delta\left(r-r^{\prime}\right) \approx \sum_{n=b, d, v, c}^{M_{i}} w_{n}^{(i)}\left(r, E_{n}^{(i)}\right) w_{n}^{(i)}\left(r^{\prime}, E_{n}^{(i)}\right),
$$

where $c$ labels the discretized scattering states from the contour $L$ and $M_{i}$ is the sum of the resonant (bound, virtual and resonant) states contained inside the the shaded area plus $N_{i}$ number of discretized continuum states. If $E_{n}^{(i)}$ is a scattering energy from the contour $L$ then the scattering state of the discretized continuum is denoted by $w_{n}^{(i)}\left(r, E_{n}^{(i)}\right)=\sqrt{h_{n}^{(i)}} u_{n}^{(i)}\left(r, E_{n}^{(i)}\right)$. If however $E_{n}^{(i)}$ corresponds to a normalized resonant state of the potential $v_{i i}(r)$ then $w_{n}^{(i)}\left(r, E_{n}^{(i)}\right)=u_{n}^{(i)}(r)$. The set of Berggren vectors form a bi-orthonormal basis in the truncated space

$$
<\tilde{w}_{n}^{(i)} \mid w_{m}^{(i)}>=\delta_{n, m} .
$$

with $<\tilde{w}_{n}^{(i)}|r>=<r| w_{n}^{(i)}>=w_{n}^{(i)}\left(r, E_{n}^{(i)}\right)$.

Having fixed the Berggren basis the solution (3) is approximated in the form

$$
\psi_{i}(r)=\sum_{k=1}^{M_{i}} C_{k}^{(i)} w_{k}^{(i)}\left(r, E_{k}^{(i)}\right) \quad i=1,2 .
$$

Using Eq. (1) we get the following set of linear equations for $C_{k}^{(i)}$

$$
\begin{gathered}
\left(E_{k}^{(1)}-E\right) C_{k}^{(1)}+\sum_{m=1}^{M_{1}}\left\langle\tilde{w}_{k}^{(1)}\left|v_{12}\right| w_{m}^{(2)}\right\rangle C_{m}^{(2)}=0 \\
k=1, \ldots, M_{1}
\end{gathered}
$$
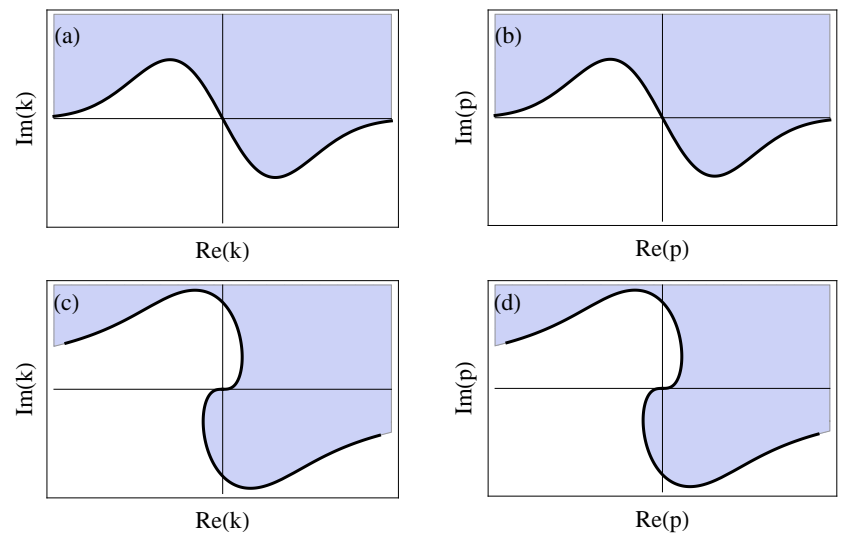

FIG. 2. (Color online) Similar to Fig. 1 but for the third Riemann sheet.

and

$$
\begin{gathered}
\left(E_{k}^{(2)}-(E-\Delta)\right) C_{k}^{(2)}+\sum_{m=1}^{M_{2}}\left\langle\tilde{w}_{k}^{(2)}\left|v_{21}\right| w_{m}^{(1)}\right\rangle C_{m}^{(1)}=0 \\
k=1, \ldots, M_{2} .
\end{gathered}
$$

These two equations can be combined into one matrix eigenvalue equation. By diagonalizing the matrix of the Hamiltonian we get complex eigenvalues $E_{\nu} \nu=$ $1, \ldots M_{1}+M_{2}$. Some complex/real eigenvalues $E_{\nu}$ can be identified as resonant states of the CC problem. The identification in this case is easy because we should find the $E_{\nu}$ eigenvalue being closest to the exact value. In general this task is more complicated, some methods can be found in Ref.[36].

In two-channel case we have a Riemann surface with four sheets. Let us define the four Riemann sheets in terms of the sign of the imaginary parts of the $k$ and the $p$ wave numbers. The Riemann sheets can be labeled by a two-term sign string $(\operatorname{sgn}(\operatorname{Im} k), \operatorname{sgn}(\operatorname{Im} p))$. We follow the standard notations introduced in Refs. [11, 15]. The first sheet is the physical one and it is signed by $(+,+)$. The second sheet is $(-,+)$ and these two levels are connected if $0<E<\Delta$. The third and fourth sheets are identified by $(-,-)$ and $(+,-)$, respectively. These two sheets are also connected if $0<E<\Delta$. If the energy $E$ is above the threshold $\Delta$ the topological structure changes: sheets one and three as well as two and four are connected [15]. The location of a resonant state determines the asymptotic behavior of its wave function. Bound state from the first Riemann sheet has square integrable wave functions in both channels. However a resonant state from the second Riemann sheet have such a wave functions that the first component asymptotically diverges and the second channel has bound state type behavior. For resonant states from the third Riemann level both components of the wave function diverge asymptotically.

When we diagonalize the CC Cox potential in Berggren bases sometimes we have to take different contours in the complex $k$ and $p$ planes in order to determine the solutions we are interested in. As we discussed earlier 
the shape of the complex contour $L$ determines which resonant states of the potential $v_{i i}(r)$ should be included into the Berggren basis of the given channel. However the shape of the contours also determines the Riemann sheets and we are able to find only the resonant $\mathrm{CC}$ states on that Riemann sheets. The $\mathrm{CC}$ resonant states of a given calculation are in the shaded areas both in the $k$ and $p$ planes.

If both the $k$ and the $p$ contours remain on the real axis we can find only bound states on the physical $(+,+)$ sheet. In order to locate resonant states on the second Riemann sheet $(-,+)$ we have to use contours of the form displayed on Fig. 1. Contours similar to the upper part ((a) and (b))can be used only for calculation of resonance states of the second Riemann sheet and for bound states of the first Riemann sheet. If a virtual state is located on the second Riemann level then the contours have to look like as displayed on the lower part ((c) and (d)) of Fig. 11 Of course, also resonance states on the second Riemann sheet can be calculated using contours similar to the lower part ((c) and (d)) of Fig. 1]. This type of contours however discard some $\mathrm{CC}$ bound state from the first Riemann level.

If resonant states located on the third Riemann sheet are to be determined, the shape of the contours depicted in Fig. 2 have to be used. Only resonance states can be determined by contours similar to the upper part ((a) and (b) of Fig. 2. The lower part is appropriate for calculation aimed at obtaining virtual states and resonance states located on the third Riemann level. We mention that using contours corresponding to the upper part ((a) and (b)) of Fig. 2 bound and resonance states of all Riemann sheets can be determined simultaneously. However numerically it is favorable to use simpler contours. For resonant state on the second Riemann sheet the accuracy of the numerical calculation is better for contours on Fig. 1 than for contours of Fig. 2. Simpler contours for states located on the fourth Riemann sheet can be similarly constructed.

\section{APPLICATION: COX POTENTIAL}

At certain parameters of the Cox potential the two channels decouple. Since the eigenvalue problem is exactly solvable, the accuracy of our program for solving the single-channel problem can be conveniently checked. This program integrates the differential equation in Eq.(18) numerically. It is important that we solve the single-channel problem accurately, since the discrete basis states are calculated using this program. The inaccuracy of the basis states would spoil the numerical results of the CC system. Therefore we deal with the solution of the single-channel case first. Then we will consider a CC system having a resonance state above the first threshold and below the second one. The threshold $\Delta$ and $\beta$ are fixed to the values 1 and 0.1 , respectively for the applications considered here.

\section{A. Single-channel solutions}

If we take the parameter $\beta=0$ then the $\mathrm{CC}$ equation with Cox potential reduces to two uncoupled equations. In this case the zeros of the Jost determinant (7) are $k=-i \alpha_{1}$ and $p=-i \alpha_{2}$. We compare the analytical results with that of our numerical calculations in Table [1. Note that the exact value of $\operatorname{Im}(k)$ has opposite sign than the value of the parameter $\alpha_{1}$.

TABLE I. Wave numbers of the anti-bound/bound states calculated exactly and numerically for the single-channel problem with potential $v_{11}(r)$ using $\beta=0$ and $\kappa=1$.

\begin{tabular}{ccc}
\hline \hline$\alpha_{1}$ & $\operatorname{Im}(k)$ exact & $\operatorname{Im}(k)$ numerical \\
\hline 0.7 & -0.7 & -0.699528 \\
0.6 & -0.6 & -0.598954 \\
0.5 & -0.5 & -0.499935 \\
0.4 & -0.4 & -0.399984 \\
0.3 & -0.3 & -0.299996 \\
0.2 & -0.2 & -0.199998 \\
0.1 & -0.1 & -0.099998 \\
-0.1 & +0.1 & 0.100001 \\
-0.2 & +0.2 & 0.200000 \\
-0.3 & +0.3 & 0.299999 \\
-0.4 & +0.4 & 0.399999 \\
\hline \hline
\end{tabular}

To calculate the eigenvalues we used the highly reliable Fortran program ANTI [54] which is based on Ixaru's method [55] for the numerical solution of the differential equation (18). This program reproduces the exact results reasonably well in most of the cases given in Table [1 The agreements are best for the bound state cases and the anti-bound wave numbers are also reproduced well, although the deviation from the exact value has increased gradually as the $\alpha_{1}$ value has increased. In solving numerically the problem the diagonal potentials $v_{11}(r)$ and $v_{22}(r)$ are cut to zero at a reasonable large $R_{\text {max }}$. Beyond $R_{\max }$ the potential is considered to be zero. The results should be at most slightly dependent on the chosen value of $R_{\max }$. With a cut-off radius $R_{\max }=13$ our numerical result is $\operatorname{Im}(k)=-0.699528$ for $\alpha_{1}=0.7$, which deviates from the exact value in the fourth decimal digit. With the same $\alpha_{1}$ value if we changed the cut-off radius value to smaller or larger values we got slightly different $\operatorname{Im}(k)$ values. (For $R_{\max }=12$ we got $\operatorname{Im}(k)=-0.700028$. For $R_{\max }=14$ we got $\operatorname{Im}(k)=-0.698944$.) So we found that the wave number of the anti-bound state depends only weakly on the cut-off radius of the diagonal potential. This is in agreement with the finding in Ref. 56 for a cut-off Woods-Saxon potential. The pole energy of the $S$-matrix is determined from the condition that the logarithmic derivatives of the internal and the external solutions of the equation (18) are equal at a matching distance $R_{\text {match}}$. See e.g. Ref. [54, 56, 57]. The internal solution is regular in $r=0$, while the external solution 
is a purely outgoing wave at $R_{\max }$. In principle the pole energy should not depend on $R_{\text {match }}$. In our calculation the value of $R_{\text {match }}$ influenced only the fifth decimal digit of $\operatorname{Im}(k)$ if we used a value in the range $R_{\text {match }} \in[1,5]$.

These comparisons of the exact and approximate energies give some hint on the limits of accuracy we can expect between the exact and approximate results of the CC calculations. We certainly can not expect better agreement for the CC case than we got for the singlechannel case.

\section{B. Coupled-channel: exact solutions}

In order to obtain the exact solution to the $\mathrm{CC}$ problem we will appeal to the inverse procedure introduced in section IIB Let us consider a resonance solution of the Cox potential with complex energy $E_{r}-i E_{i}$ so that $0<$ $E_{r}<\Delta$ and $E_{i}>0$. We will determine $\left(k_{1}, p_{1}\right)$ and $\left(k_{2}, p_{2}\right)$ from the complex energy solutions $E_{1}=E_{r}-i E_{i}$ and $E_{2}=E_{1}^{*}=E_{r}+i E_{i}$, which correspond to the wave numbers $k_{1}=k_{r}+i k_{i}$ and $k_{2}=-k_{1}^{*}=-k_{r}+i k_{i}$ with $k_{r}>0$ and $k_{i}<0$. The relations between the real and imaginary parts of the energy and wave numbers are

$$
\begin{aligned}
& k_{r}=\frac{1}{\sqrt{2}}\left[E_{r}+\sqrt{E_{r}^{2}+E_{i}^{2}}\right]^{1 / 2} \\
& k_{i}=-\frac{E_{i}}{\sqrt{2}}\left[E_{r}+\sqrt{E_{r}^{2}+E_{i}^{2}}\right]^{-1 / 2}
\end{aligned}
$$

(note that Eq. (52) of Ref. [48] is wrong). Using the threshold condition $k^{2}-p^{2}=\Delta$ we can determine $p_{r}$ and $p_{i}$ with $p_{1}=p_{r}+i p_{i}$ and $p_{2}=-p_{1}^{*}=-p_{r}+i p_{i}$. The sign of $p_{r}$ is determined by noticing that from Eq. (31) of Ref. [48] we can get $k_{r} p_{r}<0$, while the sign of $p_{i}$ is determined by the condition $p_{r} p_{i}<0$ for $E_{i}>0$ (which also implies $k_{i} p_{i}<0$ ). Considering these restrictions we have

$$
\begin{aligned}
& p_{r}=\frac{-1}{\sqrt{2}}\left[-\left(\Delta-E_{r}\right)+\sqrt{\left(\Delta-E_{r}\right)^{2}+E_{i}^{2}}\right]^{1 / 2} \\
& p_{i}=\frac{E_{i}}{\sqrt{2}}\left[-\left(\Delta-E_{r}\right)+\sqrt{\left(\Delta-E_{r}\right)^{2}+E_{i}^{2}}\right]^{-1 / 2}
\end{aligned}
$$

Taking for example $E_{r}=0.4$ and $E_{i}=0.01$ as in [48] we get $k_{r}=0.632550, k_{i}=-0.007905$ and $p_{r}=$ $-0.006455 p_{i}=0.774624$. The exact solutions for the upper sign in Eqs. (14 17) are displayed in Table II Now the CC problem has two resonances and two antibound states. According to Table II the resonances $E_{1}$ and $E_{2}$ are located in the second Riemann sheet. The anti-bound $E_{3}$ state is on the second sheet too while the second anti-bound solution $E_{4}$ is a shadow pole on the third Riemann sheet.
TABLE II. Exact energies $\left(E_{j}\right.$ for $\left.j=1, \ldots, 4\right)$ and corresponding wave numbers in the CC Cox potential with parameters given in the text.

\begin{tabular}{ccc}
\hline \hline$E_{j}$ & $k_{j}$ & $p_{j}$ \\
\hline $0.4-\mathrm{i} 0.1$ & $0.632550-\mathrm{i} 0.007905$ & $-0.006455+\mathrm{i} 0.774624$ \\
$0.4+\mathrm{i} 0.1$ & $-0.632550-\mathrm{i} 0.007905$ & $0.006455+\mathrm{i} 0.774624$ \\
-0.5604738 & $-\mathrm{i} 0.748648$ & $\mathrm{i} 1.24919$ \\
-0.5995714 & $-\mathrm{i} 0.774302$ & $-\mathrm{i} 1.26473$ \\
\hline \hline
\end{tabular}

\section{Coupled-channel: approximate solutions}

The approximate solutions are obtained by direct diagonalization of the Cox potential on Berggren basis as it is described in section III. For this we need to find the parameters $\alpha_{1}$ and $\alpha_{2}$ of the potential which give the energies of the exact solution of the previous section. Using Eqs. (12) and (13) and the upper signs we get $\alpha_{1}=0.769379934, \alpha_{2}=-0.766852669$.

TABLE III. Energies and wave numbers of discrete basis states in the first and in the second channel.

\begin{tabular}{ccc}
\hline \hline channel & $\operatorname{Re} E_{n}^{(i)}$ & $\operatorname{Im} k_{n}^{(i)}$ \\
\hline 1 & -0.573887 & -0.757553 \\
2 & -0.592006 & 0.769419 \\
\hline \hline
\end{tabular}

The solutions of CC equations using Berggren bases are carried out as follows. Two Berggren bases are calculated using the diagonal potentials $v_{11}(r)$ and $v_{22}(r)$, respectively, with the $\kappa=1$ parameter value. The parameter $\kappa$ affects the shape of the radial potentials but does not affect the CC eigenenergies. For $\kappa=1$ we found that the unperturbed potential $v_{11}(r)$ has an anti-bound state and the unperturbed potential $v_{22}(r)$ has a bound state. The actual values of the energies and wave numbers of the resonant basis states are given in Table III.

The decaying $\mathrm{CC}$ resonance state at the energy $E_{1}=$ $0.4-i 0.01$ is on the second Riemann sheet . The shape of the contour therefore should look like as the upper part ((a) and (b)) of Fig. 1] since the contour should go below $k_{1}$ and $k_{1}$ should be in the shaded area.

The basis in the first channel has no bound state therefore it is formed from the complex continuum states only. In the second channel the basis contains the unperturbed bound state and a continuum which can be taken along the real $p$-axis. Diagonalizing the Cox potential using these bases, we got a decaying resonance at the energy $E=0.400047-i 0.0100011$. The corresponding wave function is displayed in Fig. 3. The form of the wave function follows from the rules discussed in section III. The real and the imaginary parts of the first channel wave function show resonant behavior, i.e. they both diverge asymptotically. The real and the imaginary parts of the second channel wave function however both falls 
asymptotically as a bound state wave function does.

Beside the resonance states at $E_{1}$ and $E_{2}=E_{1}^{*}$ there is an anti-bound state at the energy $E_{3}=-0.5604738$. It is also in the second Riemann sheet. In order to calculate this state the contour should be taken similar to the one in the lower part ((c) and $(d))$ of the Fig. 1, since $k_{3}$ should be in the shaded area therefore we had to modify the contour used before. We still have two possibilities for selecting the basis in this channel. If the contour crosses the imaginary $k$-axis far from the origin, say at $k=(0,-1.2)$ then the anti-bound basis state will be in the shaded area and should be included into the Berggren basis. Therefore the Berggren basis in the first channel contains the unperturbed anti-bound state at $k_{n}=(0,-0.757553)$ and a set of discretized complex $k$ scattering states and in the second channel the unperturbed bound state and the real $p$ scattering states are in the basis. If we use this basis the diagonalization of the Cox potential gives a $\mathrm{CC}$ virtual state at energy $-0.561467-i 0.494 \times 10^{-7}$ which is very close to the exact $E_{3}$ value. The other option for choosing the contour is that we cross the imaginary $k$-axis just between the exact $k_{3}=(0,-0.749648)$ and the unperturbed anti-bound state at $k_{n}=(0,-0.757553)$. If we cross the imaginary axis at $(0,-0.75)$ then the unperturbed anti-bound state will be outside the shaded area therefore it won't be included in the basis. By using this basis in the first channel (the basis in the second channel remains unchanged) the diagonalization gives a $\mathrm{CC}$ virtual state at energy $-0.561008-i 0.413 \times 10^{-3}$ which is also very close to the exact $E_{3}$ value. This later basis shows an example for a case in which a correlated anti-bound state is produced by diagonalization with Berggren bases in which only bound state and complex scattering states are included. The small imaginary parts of the energies in the results of the diagonalization in both cases are due to the numerical errors of the numerical procedures used. They are beyond the accuracies of the errors of the single channel calculation of the anti-bound basis state for $\alpha_{1}=0.8$.

In the Cox potential there is an another anti-bound solution at the energy $E_{4}=-0.599544$. This state lies on the third Riemann sheet so it is a shadow pole. To be able to expand this state we have to use a contour which is similar to the one in the lower part ((c) and (d)) of Fig. 2. The Berggren basis in the first channel contains the unperturbed anti-bound state. Because of the symmetry requirement of the complex $L$ contour to the origin, in the second channel the unperturbed bound state should be excluded from the basis. Now we have no alternative in choosing the contour since $k_{4}=(0,-0.774302)$ lies lower than the negative of the imaginary part of the bound state at $-k_{n}^{(2)}=-0.769419$. The bound pole now is in the not shadowed part. But a finite number of discretized scattering states with complex $p$ wave number are naturally included in the basis. The diagonalization of the Cox potential in these bases gives an anti-bound CC state at the energy $-0.600357-i 0.149 \times 10^{-4}$, which
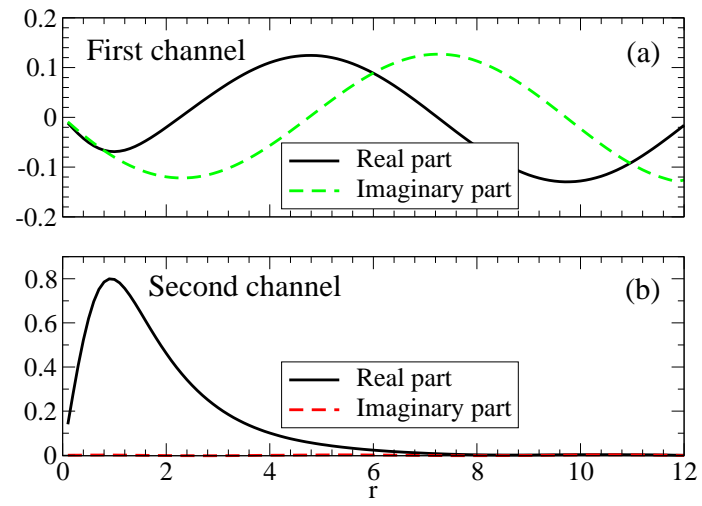

FIG. 3. The wave function of the first resonance state located in the second Riemann sheet and calculated with the Berggren expansion method. The upper (a) and lower (b) parts show the first and second channel components, respectively. The imaginary part of the second component is practically zero.

is very close to the exact $E_{4}$ value. The deviation from the exact value is again within the accuracy of reproducing the exact single particle basis states.

The Berggren basis allows the calculation of more than one resonant states simultaneously with a properly chosen basis. As an interesting example we consider the first resonance state from the second Riemann sheet and the shadow anti-bound state from the third Riemann sheet. Numerically we will show that although the states are on different sheets they can be calculated simultaneously by using the same bases. Because we want to calculate an anti-bound state in the third Riemann sheet we have to use contours similar to the one in the lower part ((c) and (d)) of Fig. 2. As we discussed before these contours however might exclude some resonance states located in the second Riemann sheet. Therefore we have to choose the crossing points of the contours and the positive imaginary axes carefully, since the wave numbers of the resonance $\mathrm{CC}$ state should be in the shaded area of Fig. 2. The Berggren basis in the first channel is formed by the unperturbed anti-bound state and a set of scattering states along the $k$ contour, while in the second channel the basis is composed of the unperturbed bound state and a set of complex $p$ scattering states. The diagonalization of the Cox potential using these bases gives the following eigenenergies $0.400214-i 0.0098868$ and $-0.599100-i 0.501 \times 10^{-3}$ simultaneously. Although these numerical results received by these bases are still quite close to the exact values of $E_{1}$ and $E_{4}$, the quality of the approximation is a little bit poorer than the ones we presented earlier with bases adjusted to the energies individually. Nevertheless the accuracies are still inside the ones of the single channels basis states. 


\section{APPLICATION TO NUCLEAR PHYSICS: A SHADOW POLE IN ${ }^{5} \mathrm{HE}$}

In this section we are going to study the structure of the $J^{\pi}=3 / 2^{+}$state of the ${ }^{5} \mathrm{He}$ nucleus in the twochannel model [58]. Here the CC Schrödinger equations are given by

$$
\begin{aligned}
& {\left[h_{1}-\left(E-\Delta_{1}\right)\right] u_{1}(r)+V_{12} u_{2}(r)=0} \\
& {\left[h_{2}-\left(E-\Delta_{2}\right)\right] u_{2}(r)+V_{12} u_{1}(r)=0}
\end{aligned}
$$

where channel one describes the ${ }^{4} \mathrm{He}-n$ partition, and channel two describes the ${ }^{3} \mathrm{H}-d$ partition. For thresholds we take $\Delta_{1}=0$, and $\Delta_{2}=17.59 \mathrm{MeV}$ [59].

The two positive-parity channel states coupled through the interaction $V_{12}$ are ${ }^{2} D_{3 / 2}$ and ${ }^{4} S_{3 / 2}$, with the following single-particle Hamiltonians $h_{1}$ and $h_{2}$, respectively

$$
\begin{aligned}
& h_{1}(r)=-\frac{\hbar^{2}}{2 \mu_{1}} \frac{d^{2}}{d r^{2}}+\frac{\hbar^{2}}{2 \mu_{1}} \frac{l_{1}\left(l_{1}+1\right)}{r^{2}}+V_{1}(r)+\bar{l}_{1} \cdot \bar{s} V_{\text {so }}(r) \\
& h_{2}(r)=-\frac{\hbar^{2}}{2 \mu_{2}} \frac{d^{2}}{d r^{2}}+V_{\text {coul }}+V_{2}(r)+V_{\text {sws }}(r)
\end{aligned}
$$

with $l_{1}=2$, and $\mu_{1}=0.805686$ amu and $\mu_{2}=1.205288$ amu, the reduced masses of the ${ }^{4} \mathrm{He}-n$ and ${ }^{3} \mathrm{H}-d$ fragmentations, respectively.

The central potential $V_{1}(r)$ and the spin-orbit term $V_{s o}(r)$ for the first channel, unlike in Ref. [58] contain no repulsive cores,

$$
\begin{aligned}
V_{1}(r) & =\frac{-V_{1}}{1+\left(\frac{r}{a_{1}}-1\right) e^{\frac{r-R_{1}}{a_{1}}}} \\
V_{\text {so }}(r) & =\frac{a_{1}^{2}}{r} \frac{V_{s o}}{V_{1}} \frac{d V_{1}(r)}{d r}
\end{aligned}
$$

with $V_{1}=70.13 \mathrm{MeV}, V_{\text {so }}=15.0 \mathrm{MeV}, a_{1}=0.85 \mathrm{fm}$, and $R_{1}=1.70 \mathrm{fm}$. Since we droped the hard core, we had to adjust the strength $V_{1}$ in order to reproduce the resonance parameters $E_{r}=0.798 \mathrm{MeV}$, and $\Gamma=0.648 \mathrm{MeV}$ of ground state $3 / 2^{-}$of ${ }^{5} \mathrm{He}$. Using these parameters, the resonant ground state is found at $\varepsilon\left(p_{3 / 2}\right)=0.799-i 0.361 \mathrm{MeV}$. The energy of the state $d_{3 / 2}$ is found at $\varepsilon\left(d_{3 / 2}\right)=15.4-i 28.0 \mathrm{MeV}$, which is a physically irrelevant resonance since the imaginary part of the energy is bigger than its real part. Since the Hamiltonian $h_{1}(r)$ does not hold any bound state or any narrow resonance, the single-particle representation of the first channel will be formed from real or complex energy scattering states exclusively.

The second channel mean-field differs from $V_{2}(r)$ used in Ref. 58 by the presence of the Coulomb interaction $V_{\text {coul }}(r)$ between the deuteron and triton and the central term having a surface Woods-Saxon (WS) form $V_{\text {sws }}(r)$,

$$
\begin{aligned}
V_{2}(r) & =\frac{-V_{2}}{1+\left(\frac{R_{2}}{a_{2}}-1\right) e^{\frac{r-R_{2}}{a_{2}}}} \\
V_{\text {sws }}(r) & =\frac{-4 V_{\text {sws }} e^{\frac{r-R_{2}}{a_{2}}}}{\left(1+e^{\frac{r-R_{2}}{a_{2}}}\right)^{2}} \\
V_{\text {coul }}(r) & =\frac{e^{2}}{r}
\end{aligned}
$$

with $V_{2}=52 \mathrm{MeV}, a_{2}=0.85 \mathrm{fm}, R_{2}=1.25 \mathrm{fm}$ and $e^{2}=1.43996508 \mathrm{MeVfm}$. The strength of the surface WS was adjusted in order to have a resonance in the partial wave $s_{1 / 2}$. In our calculation we have taken $V_{\text {sws }}=25$ $\mathrm{MeV}$ for which, the resonant energy is located $\varepsilon\left(s_{1 / 2}\right)=$ $0.125-i 0.101 \mathrm{MeV}\left(k=0.0909-i 0.0319 \mathrm{fm}^{-1}\right)$. Then, the second channel model space is formed either from real energy scattering states or from a resonance and the appropriate complex contour.

The coupling interaction $V_{12}$ between the two channels is taken as in Ref. [58],

$$
V_{12}(r)=\frac{-V_{12}}{1+\left(\frac{r}{a_{12}}-1\right) e^{\frac{r-R_{12}}{a_{12}}}}
$$

with $a_{12}=0.85 \mathrm{fm}, R_{12}=1.0 \mathrm{fm}$, and the coupling strength $V_{12}$ is a free parameter.

To study the poles of the $\mathrm{CC}$ model of ${ }^{5} \mathrm{He}$ we use complex contours in the $k$ (first channel) and $p$ (second channel) planes. Table IV gives the vertices and the number of mesh points for each segment of the complex contour. When real contour is used, the imaginary component of $k_{i}$ or $p_{i}$ is zero. The $L^{+}$contour is the collection of the line segments between the vertex points $k_{i}$ and $k_{i+1}$. There are $N_{i}$ mesh points in the segment determined by $k_{i}$ and $k_{i+1}$. The vertices were chosen in order to uncover the energy region delimited by a polygon with vertices (in MeV) $(0,0),(0,-0.5),(20,-0.5),(20,0)$ of the first channel and an identical shape shifted by $\Delta_{2}$ in the second channel.

TABLE IV. Vertices $\left(k_{i}\right.$ and $\left.p_{i}\right)$ and number of mesh points $N_{i}$ for each segment in the wave number complex plane. For further explanation see the text.

\begin{tabular}{r|ll|ll}
\hline \hline $\mathrm{i}$ & \multicolumn{1}{|c|}{$k_{i}\left(\mathrm{fm}^{-1}\right)$} & $N_{i}$ & $p_{i}\left(\mathrm{fm}^{-1}\right)$ & $N_{i}$ \\
\hline 1 & $(0,0)$ & 100 & $(0,0)$ & 100 \\
2 & $(0.09816,-0.09816)$ & 50 & $(0.1201,-0.1201)$ & 50 \\
3 & $(0.8781,-0.01097)$ & 40 & $(1.0739,-0.01342)$ & 40 \\
4 & $(1.2416,0)$ & 40 & $(1.5186,0)$ & 40 \\
5 & $(1.9631,0)$ & 40 & $(2.4011,0)$ & 40 \\
6 & $(8,0)$ & & $(8,0)$ & \\
\hline \hline
\end{tabular}

First, we solved the coupled equations in a model space formed by the real energy scattering states in the first channel and use a complex contour in the second channel 


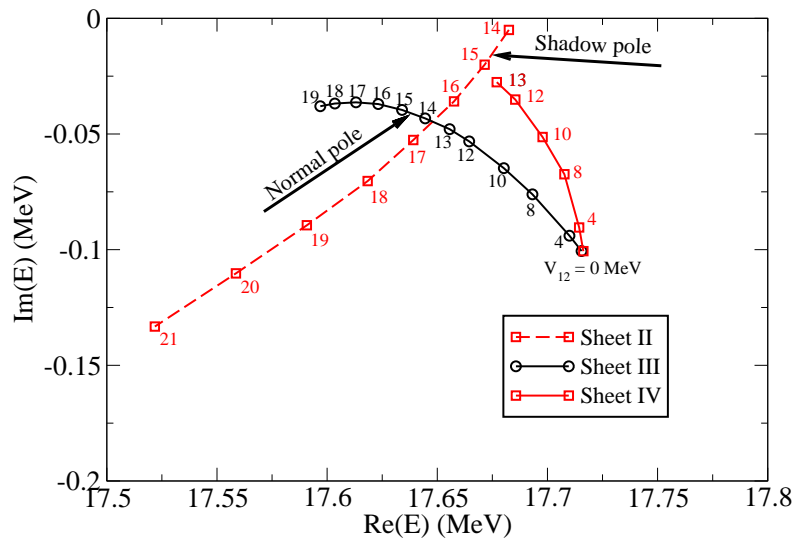

FIG. 4. (Color online) Trajectories of the physical and shadow poles as functions of the interaction strength $V_{12}$. Circles (squares) denotes the physical (shadow) poles. The numbers next to the symbols indicate the values of $V_{12}$ in $\mathrm{MeV}$. Arrows point to the positions of the physical and shadow poles at the experimental strength $V_{12}=14.7 \mathrm{MeV}$ (see details in the text)

plus the $s_{1 / 2}$ resonance state. In this way poles in the Riemann sheets $(+,+)$ or $(+,-)$ can be obtained. As the $V_{12}$ strength is increased from zero, we found that a pole starting from $E=\varepsilon\left(s_{1 / 2}\right)+\Delta_{2}$, moves upwards. We were not able to follow this pole beyond $V_{12} \simeq 13 \mathrm{MeV}$. When we use a complex contour for the first channel model space and the real energy contour for the second channel (poles of the Riemann sheets $(+,+)$ or $(-,+)$ can be obtained) then for small $V_{12}$ values we do not find any poles until we reach $V_{12} \simeq 14 \mathrm{MeV}$. Then a pole appears very close to the real axis, and it moves downwards as the interaction increases.

In Fig. 4 the pole trajectories are shown as functions of the parameter $V_{12}$. Open squares connected by a full line are the results we got with the first model space and the poles are connected by dashed lines, for second model spaces. In the the first model space the energy region of the fourth Riemann sheet is uncovered, while the second model space the energy region of the second Riemann sheet is uncovered.

According to Ref. [14], if there is a resonant pole in any of the channels with no coupling, in the CC problem this pole will generate two poles in different Riemann sheets. In our model this implies that, when the channel interaction $V_{12}$ is gradually turned on, one pole will appear in the fourth sheet $(\operatorname{Im}(\mathrm{k})>0, \operatorname{Im}(\mathrm{p})<0)$ and the second pole in the third sheet $(\operatorname{Im}(\mathrm{k})<0, \operatorname{Im}(\mathrm{p})<0)$. We have already found and discussed the movement of this latter one. When the complex contours are used in both channels (note that also the resonance must be included), we got a different resonance pole. This pole starts from the same position $\varepsilon\left(s_{1 / 2}\right)+\Delta_{2}$ as the shadow pole for $V_{12}=0$ and moves continuously toward the threshold $\Delta_{2}=17.59$ $\mathrm{MeV}$. Since this model space is characterized by $\operatorname{Im}(\mathrm{k})<0$, $\operatorname{Im}(\mathrm{p})<0$ it uncovers the fourth Riemann sheet, this pole corresponds to the physical resonance which appears in the sheet $U_{n d}$ of Ref. [20]. The movement of this pole is also depicted in 4 .

The behaviour of the pole trajectories displayed in Fig. 4 clearly shows that, as the coupling strength increases, the shadow pole moves from the fourth Riemann sheet to the second one. The same type of pole migration was observed in [31] where a microscopic cluster model was used for the description of the $J^{\pi}=3 / 2^{+}$state. From the pole trajectories we can notice that the real part of the energy of the shadow pole is always larger than that of the normal resonance pole if $0<V_{12}<19 \mathrm{MeV}$. This finding is in agreement with the result of the work 31].

The experimental energy of the normal pole is found to be at $0.048 \mathrm{MeV}$ [59] with respect to the ${ }^{3} \mathrm{H}-d$ threshold and its width is $0.0745 \mathrm{MeV}$. In our simple model calculation we found that this pole position occurs in the third sheet for $V_{12}=14.7 \mathrm{MeV}$. At this strength the normal pole in our model is found at $0.048-i 0.041 \mathrm{MeV}$, i.e. $\Gamma=0.082 \mathrm{MeV}$ in good agreement with the experimental value. At the same strength, the shadow pole is sitting in the second Riemann sheet with the following resonant parameters: $\varepsilon=0.088 \mathrm{MeV}$ and $\Gamma=0.042$ $\mathrm{MeV}$. The position of the shadow pole is close to that in other model calculations $(\varepsilon \approx 0.082 \mathrm{MeV}, \Gamma \approx 0.007$ $\mathrm{MeV})$ [20, 22] the width however is overestimated.

\section{CONCLUSIONS}

We have considered the exactly solvable CC problem of the Cox potential and showed that by using the Berggren expansion method we are able to reproduce all poles of the S-matrix even the shadow poles in agreement with the exact calculation. The proper choice of the complex contours is very important since it determines which CC states can be calculated using Berggren's basis. With suitably chosen contours we were able to calculate poles on different Riemann sheets simultaneously. We also gave a numerical example in which an anti-bound state was calculated using Berggren's basis formed only from bound and complex energy scattering states. The deviations of the numerical CC results from the exact results are within the accuracies of the calculations of the singlechannel basis states.

We have shown a nuclear physics example where a shadow pole plays an important role. In the $t+d \rightarrow \alpha+n$ fusion reaction the $\frac{3}{2}^{+}$resonance state of the nucleus ${ }^{5} \mathrm{He}$ is very important. We studied this state in a phenomenological two-channel model. In this example the emergence of two poles was observed, which are originating from a resonance in the $d+t$ channel. It was found that a shadow pole migrates between Riemann sheets if the coupling strength is varied and that at the physical strength, the normal and shadow pole parameters agree with previous findings. 


\section{ACKNOWLEDGMENTS}

Authors are grateful to Profs. B. Gyarmati, R. G. Lovas and A. Csótó for valuable discussions. This work was supported by the National Council of Research PIP-625 (CONICET, Argentina) and by the Hungarian Scientific Research Fund-OTKA K112962.

[1] A. T. Kruppa and W. Nazarewicz, Phys. Rev. C 69, 054311 (2004).

[2] A. T. Kruppa, B. Barmore, W. Nazarewicz, and T. Vertse, Phys. Rev. Lett. 84, 4549 (2000).

[3] I. J. Thompson and F. Nunes, Nuclear Reactions for Astrophysics Principles, Calculation and Applications of Low-Energy Reactions (Cambridge University Press, England, 2009).

[4] B. Marcelis, E. van Kempen, B. Verhaaar, and S. M. Kokkelmans, Phys. Rev. A 70, 012701 (2004).

[5] N. Nygard, B. Schneider, and P. Julienne, Phys. Rev. A 73, 042705 (2006).

[6] A. Cieplý and J. Smejkal, Nucl. Phys. A 919, 46 (2013), arXiv:1308.4300v2 [hep-ph]

[7] A. Doté, T. Inoue, and T. Myo, Nucl. Phys. A 912, 66 (2013), arXiv:1207.5279v3 [nucl-th].

[8] A. Doté, T. Inoue, and T. Myo, Phys. Rev. C 84, 204549 (2000).

[9] K. Miyagawa and H. Yamamura, Phys. Rev. C 60, 024003 (1999).

[10] R. Eden, P. Landshoff, D. Olive, and J. Polkinghorne, The Analytic S-Matrix (Cambridge University Press, Cambridge, 1966).

[11] A. M. Badalyan, L. P. Kok, M. I. Polikarpov, and Y. Simonov, Phys. Rep. 82, 31 (1982).

[12] C. Grama, N. Grama, and I. Zamfirescu, Phys. Rev. A 61, 032716 (2000).

[13] C. Grama, N. Grama, and I. Zamfirescu, Phys. Rev. A 68, 032723 (2003).

[14] R. J. Eden and J. R. Taylor, Phys. Rev. B 133, 1575 (1964).

[15] W. R. Frazer and A. W. Hendry, Phys. Rev B 134, 1307 (1964).

[16] W. van Dijk, K. Spyksma, and M. West, Phys. Rev. A 78, 022108 (2008).

[17] S. A. Rakityansky and N. Elander, Int. J. of Quant. Chem. 106, 1105 (2006).

[18] R. M. Potvliege and R. Shakeshaft, Phys. Rev. A 38, 6190 (1988).

[19] M. Dorr and R. M. Potvliege, Phys. Rev. A 41, 1472 (1990).

[20] G. M. Hale, R. E. Brown, and N. Jarmie, Phys. Rev. Lett. 59, 763 (1987).

[21] L. N. Bogdanova, G. M. Hale, and V. E. Markushin, Phys. Rev. C 44, 1289 (1991).

[22] A. Csótó, R. G. Lovas, and A. T. Kruppa, Phys. Rev. Lett. 70, 1389 (1993).

[23] B. C. Pearce and B. F. Gibson, Phys. Rev. C 40, 902 (1989).

[24] D. Morgan and M. R. Pennington, Phys. Rev. Lett. 59, 2818 (1987).
[25] D. Morgan and M. R. Pennington, Phys. Lett. B 258, 444 (1991).

[26] J. P. Cannata, F.and Dedonder and L. Leśniak, Z. Phys. A 343, 451 (1992).

[27] G. Cattapan and P. Lotti, Eur. Phys. J. B 60, 182 (2007).

[28] Y. Ho, Phys. Rep. 99, 1 (1983).

[29] N. Moiseyev, Phys. Rep. 302, 212 (1998).

[30] A. Kruppa, G. Papadimitriou, W. Nazarewicz, and N. Michel, Phys. Rev. C 89, 014330 (2014).

[31] A. Csótó, Phys. Rev. A 48, 3390 (1993).

[32] R. M. Id Betan, Physics Letters B 730, 18 (2014).

[33] T. Berggren, Nucl. Phys. A 109, 265 (1968).

[34] R. Id Betan, R. J. Liotta, N. Sandulescu, and T. Vertse, Phys. Rev. Lett. 89, 042501 (2002).

[35] N. Michel, W. Nazarewicz, M. Płoszajczak, and K. Bennaceur, Phys. Rev. Lett. 89, 042502 (2002).

[36] N. Michel, W. Nazarewicz, M. Ploszajczak, and T. Vertse, J. Phys. G 36, 013101 (2009).

[37] R. Id Betan, R. J. Liotta, N. Sandulescu, and T. Vertse, Phys. Lett. B 584, 48 (2004).

[38] T. Vertse, P. Curutchet, R. Liotta, and J. Bang, Acta Phys. Hung. 65, 305 (1989).

[39] N. Michel, K. Matsuyanagi, and M. Stoitsov, Phys. Rev. C 78, 044319 (2008).

[40] R. Id Betan, A. T. Kruppa, and T. Vertse, Phys. Rev. C 78, 044308 (2008).

[41] J. R. Cox, Journal of Mathematical Physics 5, 1065 (1964).

[42] A. M. Pupasov, B. F. Samsonov, and J. M. Sparenberg, Phys. Rev. A 77, 012724 (2008).

[43] J. M. Sparenberg, B. F. Samsonov, F. Foucart, and D. Baye, J. Phys. A: Math. Gen. 39, L639 (2006).

[44] B. F. Samsonov, J. Sparenberg, and D. Baye, J. Phys. A: Math. Gen. 40, 4225 (2007).

[45] L. S. Brown and G. M. Hale, Phys. Rev. C 89, 014622 (2014).

[46] G. M. Hale, L. S. Brown, and M. W. Paris, Phys. Rev. C 89, 014622 (2014).

[47] P. Navratil and S. Quaglioni, Phys. Rev. Lett. 108, 042503 (2011).

[48] A. M. Pupasov, B. F. Samsonov, and J. Sparenberg, Phys. Rev. A 77, 012724 (2008), arXiv:0709.0343v1[quant-ph]

[49] R. G. Newton, Scattering Theory of Waves and Particles., 2nd ed. (Springer-Verlag New York Heidelberg Berlin p. 368, 1982).

[50] N. Michel, J. Math. Phys. 49, 022109 (2008).

[51] N. Michel, W. Nazarewicz, and M. Płoszajczak, Phys. Rev. C 70, 064313 (2004).

[52] A. Mukhamedzhanov and M. Akin, Eur. Phys. J. A 37, 185 (2008). 
[53] R. J. Liotta, E. Maglione, N. Sandulescu, and T. Vertse, Physics Letters B 367, 1 (1996).

[54] L. G. Ixaru, M. Rizea, and T. Vertse, Comput. Phys. Commun. 85, 217 (1995).

[55] L. G. Ixaru, Numerical Methods for Differential Equations and Applications (D. Reidel, Publ. Comp. Dordrecht, Boston, Lancaster, 1984).
[56] J. Darai, A. Rácz, and R. G. Lovas, Phys. Rev. C 86, 014314 (2012).

[57] T. Vertse, K. F. Pál, and Z. Balogh, Comput. Phys. Commun. 27, 309 (1982)

[58] B. De Facio, R. K. Umerjee, and J. L. Gammel, Phys. Rev. 151, 819 (1966).

[59] http://www.tunl.duke.edu/nucldata. 